PROCEEDINGS OF THE

AMERICAN MATHEMATICAL SOCIETY

Volume 132, Number 12, Pages 3627-3628

S 0002-9939(04)07467-2

Article electronically published on May 12, 2004

\title{
A CHARACTERIZATION OF AN ORDER IDEAL IN RIESZ SPACES
}

\author{
S. ALPAY, E. YU. EMEL'YANOV, AND Z. ERCAN \\ (Communicated by Joseph A. Ball)
}

\begin{abstract}
In this paper we give a characterization of order ideals in Riesz spaces.
\end{abstract}

Recall that an ordered vector space $E$ is a vector space with a partial order satisfying

$$
x \leq y \Longrightarrow \alpha x+z \leq \alpha y+z
$$

for all $z \in E$ and $0 \leq \alpha \in \mathbf{R}$. A partially ordered vector space $E$ is called a Riesz space if $\sup \{x, y\}$ exists for each $x, y \in E$. If $E$ is a Riesz space, then for each $x \in E$ we write

$$
x^{+}=\sup \{x, 0\}, \quad x^{-}=\sup \{-x, 0\} \quad \text { and } \quad|x|=\sup \{x,-x\} .
$$

Definition 1. A vector subspace $F$ of a Riesz space $E$ is said to be an order ideal whenever $|x| \leq|y|$ and $y \in F$ imply $x \in F$.

In the Nakano terminology an order ideal was called a semi-normal subspace. In the Soviet literature it was called normal sublineal. Although the order ideal theory plays a central role in Riesz spaces theory, to the best of our knowledge, there is no characterization of order ideal in direct terms without use of inequalities. For further details about order ideals we refer to [1], [2] and [3]. We present a characterization of an order ideal as follows:

Theorem 2. Let $F$ be a vector subspace of a Riesz space E. Then the following conditions are equivalent:

i. $|x+y|-|y| \in F$ for each $x \in F$ and $y \in E$.

ii. $|x+y|-|x-y| \in F$ for each $x \in F$ and $y \in E$.

iii. $F$ is an order ideal.

Proof. $\mathbf{i} \Longrightarrow$ ii: Let $x \in F$ and $y \in E$ be given. Then clearly

$$
|x+y|-|x-y|=(|x+y|-|y|)-(|x+(-y)|-|-y|) \in F .
$$

ii $\Longrightarrow$ iii: Let $x \in F$. Since

$$
x^{+}=\frac{1}{2}\left(\left|x+x^{+}\right|-\left|x-x^{+}\right|\right) \in F,
$$

Received by the editors July 7, 2003 and, in revised form, August 13, 2003.

2000 Mathematics Subject Classification. Primary 46A40.

Key words and phrases. Riesz spaces, order ideals.

This work was supported by the Scientific and Technical Research Council of Turkey.

(C)2004 American Mathematical Society 
$F$ is a Riesz subspace. Let $x \in E$ be given with $|x| \in F$. Then

$$
x=\left(x^{+}-x^{-}\right)=\frac{1}{2}(|| x|+x|-|| x|-x|) \in F .
$$

So, it is enough to show that $x \in F$ whenever $0 \leq x \leq y$ for some $y \in F$. Let $x \in E, y \in F$ with $0 \leq x \leq y$. Since $|y+(-x)|-|y-(-x)| \in F$ we have

$$
x=-\frac{1}{2}(y-x-(y+x))=-\frac{1}{2}(|y+(-x)|-|y-(-x)|) \in F .
$$

Hence $F$ is an ideal.

$\mathrm{iii} \Longrightarrow \mathbf{i}$ : Let $x \in F$ and $y \in E$. Then from the inequality

$$
|| x+y|-| y|| \leq|x|,
$$

we immediately have $|x+y|-|y| \in F$.

As an application of the above theorem we can reprove that the algebraic sum of two order ideals of a Riesz space is also an order ideal, as follows: Let $E$ be a Riesz space, and let $I, J$ be order ideals of $E$. Then $I+J$ is also an order ideal. Let $a \in I, b \in J$ and $x \in E$. Then

$$
|(a+b)-x|-|x|=|a+(b-x)|-|b-x|+|b-x|-|x| \in I+J .
$$

So, from the previous theorem, $I+J$ is an order ideal.

Remark. The theorem above motivates the following generalization of the notions of Riesz subspace and order ideal: Let $E$ be a Riesz space, and let $X$ and $Y$ be vector subspaces of $E$. We call $X$ an order ideal with respect to $Y$ if

$$
|x+y|-|y| \in X \quad \text { for all } \quad x \in X, y \in Y \text {. }
$$

Then $X$ is a Riesz subspace of $E$ if and only if it is an ideal with respect to $\{0\}$, and $X$ is an order ideal if and only if it is an ideal with respect to $E$.

\section{REFERENCES}

[1] W.A.J. Luxemburg and A.C. Zaanen, Riesz Spaces I, North-Holland Mathematical Library. North-Holland Publishing Co., Amsterdam-London; New York, 1971. MR 58:23483

[2] A.C. Zaanen, Ideals in Riesz spaces. Troisième Colloque sur l'Analyse Fonctionnelle (Liège, 1970), pp. 137-146. Vander, Louvain, 1971. MR 53:3640

[3] A.C. Zaanen, Introduction to operator theory in Riesz spaces, Springer-Verlag, Berlin, 1997. MR 2000c:47074

Department of Mathematics, Middle East Technical University, 06531 Ankara, TURKEY

E-mail address: safak@math.metu.edu.tr

Sobolev Institute of Mathematics, Acad. Koptyug PR. 4, 630090 Novosibirsk, Russia Current address: Department of Mathematics, Middle East Technical University, 06531 Ankara, Turkey

E-mail address: emelanov@math.nsc.ru

Department of Mathematics, Middle East Technical University, 06531 Ankara, TURKEY

E-mail address: zercan@metu.edu.tr 